\title{
ON INTEGRATION-BY-PARTS FOR WEIGHTED INTEGRALS
}

FRED M. WRIGHT AND JAMES D. BAKER

1. Introduction. In this paper we deal with a weighted refinement integral which has as special cases the mean sigma integral due to H. L. Smith [1] and studied by R. E. Lane [2], the interior refinement integral due to Dushnik [3], and the left and right Cauchy refinement integrals. This integral is related under rather general conditions to the Young refinement integral and hence to the Lebesgue-Stieltjes integral. We define our weighted refinement integral as follows.

Definition 1.1. Let $p$ be an integer such that $p \geqq 2$, and let $\left(w_{1}, w_{2}\right.$, $\left.\cdots, w_{p}\right)$ be an ordered $p$-tuple of real numbers such that $w_{1}+w_{2}$ $+\cdots+w_{p}=1$. Let $f$ and $g$ be real-valued functions on the closed interval $[a, b]$ of the real axis. For a partition

$$
P=\left\{a<x_{0}<x_{1}<\cdots<x_{n}=b\right\}
$$

of $[a, b]$, choose for each $i=1,2, \cdots, n$ a partition

$$
\Delta_{i}=\left\{x_{i-1}=\zeta_{1, i}<\zeta_{2, i}<\cdots<\zeta_{p, i}=x_{i}\right\}
$$

of $\left[x_{i-1}, x_{i}\right]$ consisting of $p$ points and form the sum

$$
S\left(P ; \Delta_{1}, \cdots, \Delta_{n}\right)=\sum_{i=1}^{n}\left[\sum_{j=1}^{p} w_{j} \cdot f\left(\zeta_{j, i}\right)\right]\left[g\left(x_{i}\right)-g\left(x_{i-1}\right)\right] .
$$

If the refinement limit

$$
\lim _{P, 5} S\left(P ; \Delta_{1}, \cdots, \Delta_{n}\right)
$$

exists and is finite, this limit will be denoted by

$$
\left[F,\left(w_{1}, w_{2}, \cdots, w_{p}\right)\right] \int_{a}^{b} f(x) d g(x)
$$

and will be called the $\left(w_{1}, w_{2}, \cdots, w_{p}\right)$-weighted refinement integral of $f$ with respect to $g$ on $[a, b]$.

In $\S 2$ we consider existence conditions for (1.5). First, we have two theorems which provide necessary conditions for the existence of (1.5) involving the behavior of $f(x)$ and $g(x)$ as $x$ approaches a point $c \in[a, b)$ from the right. Analogous results hold for limits from the left. We then consider existence conditions for (1.5) when $g$ is of

Received by the editors August 8, 1968 and, in revised form, October 5, 1968. 
bounded variation on $[a, b]$. We begin with a theorem which provides a sufficient set of existence conditions as well as a formula for (1.5) when $f$ is bounded and $g$ is a saltus function on $[a, b]$. This is an extension of R. E. Lane's Theorem 3.1 in [2]. We next consider the case where $g$ is a continuous function of bounded variation and $f$ is bounded on $[a, b]$ and present the result that the existence of (1.5) implies the existence of the Riemann-Stieltjes norm integral

$$
\operatorname{RSN} \int_{a}^{b} f(x) d g(x) .
$$

We then relate (1.5) to the Young refinement integral

$$
\text { FY } \int_{a}^{b} f(x) d g(x)
$$

when $f$ is bounded and $g$ is of bounded variation on $[a, b]$.

In $\$ 3$ we first present three theorems concerning integration-byparts for (1.5) when $p=3$ and $g$ is of bounded variation on $[a, b]$. From the third of these theorems we have the integration-by-parts type formula

$$
\mathrm{FY} \int_{a}^{b} g(x) d f(x)=f(b) \cdot g(b)-f(a) \cdot g(a)-\mathrm{Fi} \int_{a}^{b} f(x) d g(x)
$$

given by J. S. MacNerney in [4] involving the Young refinement integral

$$
\mathrm{FY} \int_{a}^{b} g(x) d f(x)
$$

and the interior refinement integral

$$
\mathrm{Fi} \int_{a}^{b} f(x) d g(x)
$$

when one of the two functions $f, g$ is of bounded variation and the other is quasi-continuous on $[a, b]$.

2. Existence conditions. Throughout this section we assume that $p$ is an integer such that $p \geqq 2$, that $\left(w_{1}, w_{2}, \cdots, w_{p}\right)$ is an ordered $p$-tuple of real numbers such that $w_{1}+w_{2}+\cdots+w_{p}=1$, and that $f$ and $g$ are real-valued functions on the closed interval $[a, b]$ of the real axis. 
We begin with the following necessary condition for the existence of (1.5).

THEOREM 2.1. Suppose $p>2$, and suppose there is an integer $\bar{j}$ satisfying $1<j<p$ such that $w_{j} \neq 0$. If there is a $c \in[a, b)$ such that $g$ is not continuous from the right at $c$ and such that $f$ does not have a finite righthand limit at c, then (1.5) does not exist.

Proof. There are positive numbers $\omega$ and $k$ such that for $0<\delta$ $<(b-c)$ there exist $x \in(c, c+\delta)$ such that $|g(x)-g(c)| \geqq \omega$ and $x^{\prime}, x^{\prime \prime} \in(c, c+\delta)$ such that $\left|f\left(x^{\prime}\right)-f\left(x^{\prime \prime}\right)\right| \geqq k$.

Let (1.1) be any partition of $[a, b]$ containing $c$. Let $x_{i-1}=c$. Let $d \in\left(c, x_{i}\right)$ such that $|g(d)-g(c)| \geqq \omega$. Let $s, t \in(c, d)$ such that $s>t$ and $|f(t)-f(s)| \geqq k$. Let $\bar{P}=\left\{a=\bar{x}_{0}<\bar{x}_{1}<\cdots<\bar{x}_{n+1}=b\right\}$ be $P$ $\cup\{d\}$. Let $\bar{\Delta}_{\bar{i}}^{(1)}$ be a partition of $\left[\bar{x}_{i-1}, \bar{x}_{i}\right]$ consisting of $p$ points with $s$ as the $j$ th point and with the $(j+1)$ st point greater than $t$. Let $\bar{\Delta}_{\bar{i}}^{(2)}$ be the partition of $\left[\bar{x}_{i-1}, \bar{x}_{i}\right]$ consisting of $p$ points such that each point except the $j$ th is the same as the corresponding point of $\bar{\Delta}_{\bar{i}}^{(1)}$ and such that $t$ is the $j$ th point. For each positive integer $i$ not exceeding $n+1$ and different from $\bar{i}$, let $\bar{\Delta}_{i}^{(1)}$ and $\bar{\Delta}_{i}^{(2)}$ be the same partition of $\left[\bar{x}_{\bar{i}-1}, \bar{x}_{i}\right]$ consisting of $p$ points. Then

$$
\begin{aligned}
\mid S\left(\bar{P} ; \bar{\Delta}_{1}^{(2)}, \bar{\Delta}_{2}^{(2)},\right. & \left.\cdots, \bar{\Delta}_{n+1}^{(2)}\right)-S\left(\bar{P} ; \bar{\Delta}_{1}^{(1)} \bar{\Delta}_{2}^{(1)}, \cdots, \bar{\Delta}_{n+1}^{(1)}\right) \mid \\
& =\left|w_{i}\right| \cdot|f(t)-f(s)| \cdot|g(d)-g(c)| \geqq\left|w_{T}\right| \cdot k \cdot \omega .
\end{aligned}
$$

Thus, (1.5) does not exist.

We also present the following necessary condition for the existence of (1.5). For brevity the proof is omitted.

Theorem 2.2. Suppose $w_{p} \neq 0$. If there is a $c \in[a, b)$ such that $g\left(c^{+}\right)$ exists and is finite, such that $g\left(c^{+}\right) \neq g(c)$, and such that $f$ does not have $a$ finite right-hand limit at $c$, then (1.5) does not exist.

We now consider existence conditions for (1.5) when $g$ is of bounded variation on $[a, b]$. First we present the following extension of Lane's Theorem 3.1 in [2] for the case where $g$ is a saltus function.

ThEOREM 2.3. Suppose $g$ is a saltus function and $f$ is bounded on $[a, b]$. Let

$$
\mathcal{S}^{+}=\left\{x \in[a, b) \mid g\left(x^{+}\right) \neq g(x)\right\}
$$

and

$$
\mathcal{S}^{-}=\{x \in(a, b] \mid g(x) \neq g(x)\} .
$$

If there is an integer $j$ satisfying $1<j \leqq p$ such that $w_{j} \neq 0$, suppose $f\left(x^{+}\right)$ 
exists for all $x \in \mathcal{S}^{+}$; if there is an integer $j$ satisfying $1 \leqq j<p$ such that $w_{j} \neq 0$, suppose $f\left(x^{-}\right)$exists for all $x \in \mathcal{S}^{-}$. Then, $(1.5)$ exists and equals

$$
\begin{aligned}
\sum_{x \in \mathcal{S}^{+}}\left[\left(1-w_{1}\right) f\left(x^{+}\right)+w_{1} f(x)\right]\left[g\left(x^{+}\right)-g(x)\right] \\
+\sum_{x \in \mathcal{S}^{-}}\left[w_{p} f(x)+\left(1-w_{p}\right) f\left(x^{-}\right)\right]\left[g(x)-g\left(x^{-}\right)\right] .
\end{aligned}
$$

Proof. Our argument is analogous to Lane's proof of his Theorem 3.1 in [2].

Next wo give a theorem for the case where $g$ is continuous and of bounded variation on $[a, b]$.

TheOREM 2.4. Suppose $g$ is continuous and of bounded variation on $[a, b], f$ is bounded on $[a, b]$, and (1.5) exists. Then the RiemannStieltjes norm integral (1.6) exists.

Proof. Our proof is patterned after the proof given by K. P. Smith and F. M. Wright of their Theorem 2.1 in [5].

From Theorems 2.1 and 2.2, their analogues for left-hand limits, and Theorems 2.3 and 2.4, we have the following result relating (1.5) and the Young refinement integral (1.7).

THEOREM 2.5. Suppose $g$ is of bounded variation on $[a, b], f$ is bounded on $[a, b]$, and (1.5) exists. Then (1.7) exists and equals

$$
\begin{aligned}
{\left[F,\left(w_{1}, w_{2} \cdots,\right.\right.} & \left.\left., w_{p}\right)\right] \int_{a}^{b} f(x) d g(x) \\
& -\left(1-w_{1}\right) \cdot \sum_{x \in[a, b)}\left[f\left(x^{+}\right)-f(x)\right]\left[g\left(x^{+}\right)-g(x)\right] \\
& +\left(1-w_{p}\right) \cdot \sum_{x \in(a, b]}\left[f(x)-f\left(x^{-}\right)\right]\left[g(x)-g\left(x^{-}\right)\right] .
\end{aligned}
$$

3. Integration-by-parts. Throughout this section we assume that $\left(w_{1}, w_{2}, w_{3}\right)$ is an ordered triple of real numbers such that $w_{1}+w_{2}+w_{3}$ $=1$ and $f$ and $g$ are real-valued functions on the closed interval $[a, b]$ of the real axis.

If $g$ is of bounded variation on $[a, b]$ we have the following result.

THEOREM 3.1. Suppose $g$ is of bounded variation and $f$ is bounded on $[a, b]$. Let

$$
\mathcal{S}^{+}=\left\{x \in[a, b) \mid g\left(x^{+}\right) \neq g(x)\right\}
$$

and

$$
\mathcal{S}^{-}=\left\{x \in(a, b] \mid g\left(x^{-}\right) \neq g(x)\right\}
$$




\section{Suppose}

$$
\left[F,\left(w_{1}, w_{2}, w_{3}\right)\right] \int_{a}^{b} f(x) d g(x)
$$

exists, $f\left(x^{+}\right)$exists for $x \in \mathcal{S}^{+}$, and $f\left(x^{-}\right)$exists for $x \in \mathcal{S}^{-}$. Then

$$
\left[F,\left(w_{1}, w_{2}, w_{3}\right)\right] \int_{a}^{b} g(x) d f(x)
$$

exists and equals

$$
\begin{aligned}
f(b) g(b)-f(a) g(a) & -\left[F,\left(w_{1}, w_{2}, w_{3}\right)\right] \int_{a}^{b} f(x) d g(x) \\
& -\left(2 w_{1}-1\right) \sum_{x \in \mathcal{S}^{+}}\left[f\left(x^{+}\right)-f(x)\right]\left[g\left(x^{+}\right)-g(x)\right] \\
& +\left(2 w_{3}-1\right) \sum_{x \in \mathcal{S}^{-}}\left[f(x)-f\left(x^{-}\right)\right]\left[g(x)-g\left(x^{-}\right)\right] .
\end{aligned}
$$

Proof. Let $s$ be a saltus function and $\psi$ a continuous function on $[a, b]$ such that $g(x)=s(x)+\psi(x)$ for all $x \in[a, b]$.

Let (1.1) be any partition of $[a, b]$, and for each $i=1,2, \cdots, n$, let $\Delta_{i}=\left\{x_{i-1}=\zeta_{1, i}<\zeta_{2,1}<\zeta_{3, i}=x_{i}\right\}$ be any partition of $\left[x_{i-1}, x_{i}\right]$ consisting of three points. Then

$$
\begin{aligned}
\sum_{i=1}^{n}[ & \left.\sum_{j=1}^{s} w_{j} s\left(\zeta_{j, i}\right)\right]\left[f\left(x_{i}\right)-f\left(x_{i-1}\right)\right] \\
& =f(b) s(b)-f(a) s(a) \\
& -\sum_{i=1}^{n}\left[\sum_{j=1}^{3} w_{j} f\left(\zeta_{j, i}\right)\right]\left[s\left(x_{i}\right)-s\left(x_{i-1}\right)\right] \\
& -\left(2 w_{1}-1\right) \sum_{i=1}^{n}\left[f\left(\zeta_{2, i}\right)-f\left(x_{i-1}\right)\right]\left[s\left(\zeta_{2, i}\right)-s\left(x_{i-1}\right)\right] \\
& +\left(2 w_{3}-1\right) \sum_{i=1}^{n}\left[f\left(x_{i}\right)-f\left(\zeta_{2, i}\right)\right]\left[s\left(x_{i}\right)-s\left(\zeta_{2, i}\right)\right] \\
& +\left(w_{1}-w_{3}\right)\left\{\sum_{i=1}^{n}\left[f\left(x_{i-1}\right)-f\left(\zeta_{2, i}\right)\right]\left[s\left(x_{i}\right)-s\left(\zeta_{2, i}\right)\right]\right. \\
& \left.+\sum_{i=1}^{n}\left[f\left(\zeta_{2, i}\right)-f\left(x_{i}\right)\right]\left[s\left(\zeta_{2, i}\right)-s\left(x_{i-1}\right)\right]\right\}
\end{aligned}
$$


Now

$$
\begin{aligned}
\lim _{P, \supset} \sum_{i=1}^{n} f\left(\zeta_{2, i}\right) & {\left[s\left(\zeta_{2, i}\right)-s\left(x_{i-1}\right)\right] } \\
& =\sum_{x \in \delta^{+}} f\left(x^{+}\right)\left[s\left(x^{+}\right)-s(x)\right] \\
& =\lim _{P, \supset} \sum_{i=1}^{n} f\left(x_{i}\right)\left[s\left(\zeta_{2, i}\right)-s\left(x_{i-1}\right)\right]
\end{aligned}
$$

and

$$
\begin{aligned}
\lim _{P, D} & \sum_{i=1}^{n} f\left(x_{i-1}\right)\left[s\left(\zeta_{2, i}\right)-s\left(x_{i-1}\right)\right] \\
& =\sum_{x \in \mathrm{S}^{+}} f(x)\left[s\left(x^{+}\right)-s(x)\right]
\end{aligned}
$$

analogous observations hold for left-hand limits. Therefore

$$
\left[F,\left(w_{1}, w_{2}, w_{3}\right)\right] \int_{a}^{b} s(x) d f(x)
$$

exists and equals

$$
\begin{aligned}
f(b) s(b) & -f(a) s(a)-\left[F,\left(w_{1}, w_{2}, w_{3}\right)\right] \int_{a}^{b} f(x) d s(x) \\
& -\left(2 w_{1}-1\right) \sum_{x \in \delta^{+}}\left[f\left(x^{+}\right)-f(x)\right]\left[g\left(x^{+}\right)-g(x)\right] \\
& +\left(2 w_{3}-1\right) \sum_{x \in S^{-}}\left[f(x)-f\left(x^{-}\right)\right]\left[g(x)-g\left(x^{-}\right)\right] .
\end{aligned}
$$

The Riemann-Stieltjes norm integral RSN $\int_{a}^{b} f(x) d \psi(x)$ exists. Thus, $\operatorname{RSN} \int_{a}^{b} \psi(x) d f(x)$ exists and equals

$$
f(b) \psi(b)-f(a) \psi(a)-\operatorname{RSN} \int_{a}^{b} f(x) d \psi(x) .
$$

Therefore (3.2) exists and (3.3) holds.

The result on integration-by-parts for the Lebesgue-Stieltjes integral given by H. Schärf in [6] follows from Theorems 3.1 and 2.5.

Using a proof patterned after our proof of Theorem 3.1, we have the following result.

Theorem 3.2. Suppose (3.1) exists. If $w_{2}=0$, then 


$$
\left[F,\left(w_{3}, w_{2}, w_{1}\right)\right] \int_{a}^{b} g(x) d f(x)
$$

exists and equals

$$
f(b) g(b)-f(a) g(a)-\left[F,\left(w_{1}, w_{2} w_{3}\right)\right] \int_{a}^{b} f(x) d g(x) .
$$

If $f$ and $g$ satisfy the hypotheses of Theorem 3.1, then (3.4) exists and equals

$$
\begin{aligned}
& f(b) g(b)-f(a) g(a)-\left[F,\left(w_{1}, w_{2}, w_{3}\right)\right] \int_{a}^{b} f(x) d g(x) \\
&+w_{2}\left\{\sum_{x \in \mathcal{S}^{+}}\left[f\left(x^{+}\right)-f(x)\right]\left[g\left(x^{+}\right)-g(x)\right]\right. \\
&\left.\quad-\sum_{x \in \mathcal{S}^{-}}\left[f(x)-f\left(x^{-}\right)\right]\left[g(x)-g\left(x^{-}\right)\right]\right\} .
\end{aligned}
$$

The following result provides a quite simple integration-by-parts type formula and can also be established by using a proof patterned after our proof of Theorem 3.1.

THEOREM 3.3. Suppose (3.1) exists. If $w_{2}=0$, then

$$
\left[F,\left(1-w_{1},-w_{2}, \mathbf{1}-w_{3}\right)\right] \int_{a}^{b} g(x) d f(x)
$$

exists and equals (3.5). If $f$ and $g$ satisfy the hypotheses of Theorem 3.1, then (3.7) exists and equals (3.5).

The next result provides a necessary condition for the existence of (3.1) when $f$ is continuous and of bounded variation and $g$ is bounded on $[a, b]$.

THEOREM 3.4. Suppose $f$ is continuous and of bounded variation and $g$ is bounded on $[a, b]$. Suppose (3.1) exists. Then the Riemann-Stieltjes norm integral

$$
\operatorname{RSN} \int_{a}^{b} g(x) d f(x)
$$

exists.

Proof. If $w_{2}=0$, we have from Theorem 3.2 that (3.4) exists, so 
(3.8) exists in view of Theorem 2.4. If $w_{2} \neq 0$, our proof that (3.8) exists is patterned after the proof given by K. P. Smith and F. M. Wright of their Theorem 2.1 in [5]. Thus (3.8) exists.

From Theorems 2.3, 3.1, 3.2, 3.3, and 3.4 we have the following result concerning the existence of (3.1) when $f$ is of bounded variation on $[a, b]$.

THeOREM 3.5. Suppose $f$ is of bounded variation and $g$ is bounded on $[a, b]$. Suppose that $g\left(x^{+}\right)$exists for all $x \in[a, b)$ such that $f\left(x^{+}\right) \neq f(x)$ and that $g\left(x^{-}\right)$exists for all $x \in(a, b]$ such that $f\left(x^{-}\right) \neq f(x)$. Finally, suppose (3.1) exists. Then (3.2), (3.4), and (3.7) exist.

From Theorem 3.3 we have the integration-by-parts type formula (1.8) given by J. S. MacNerney in [4] involving the Young refinement integral (1.9) and the interior refinement integral (1.10) when one of the two functions $f, g$ is of bounded variation and the other is quasi-continuous on $[a, b]$. In order to see this, we need Theorem 2.5 and the following result.

THEOREM 3.6. Suppose $f$ is quasi-continuous and $g$ is of bounded variation on $[a, b]$. Then the Young refinement integral (1.9) and the weighted refinement integral

$$
[F,(1,-1,1)] \int_{a}^{b} g(x) d f(x)
$$

exist and are equal.

Proof. Suppose $\epsilon>0$. Let $M$ be a positive real number such that $|f(x)| \leqq M$ for $x \in[a, b]$. Let $\bar{P}=\left\{a=\bar{x}_{0}<\bar{x}_{1}<\cdots<\bar{x}_{\tilde{n}}=b\right\}$ be a partition of $[a, b]$ such that, for each $k=1,2, \cdots, \bar{n}$,

$$
\left|f\left(x^{\prime}\right)-f\left(x^{\prime \prime}\right)\right|<\frac{\epsilon}{2\{V(g,[a, b])+1\}}
$$

for $x^{\prime}, x^{\prime \prime} \in\left(\bar{x}_{k-1}, \bar{x}_{k}\right)$. For each $k=1,2, \cdots, \bar{n}$, suppose $y_{k}^{\prime} \in\left(\bar{x}_{k-1}, \bar{x}_{k}\right)$ such that $\left|g\left(x^{\prime}\right)-g\left(x^{\prime \prime}\right)\right|<\epsilon / 8 \bar{n} M$ for $x^{\prime}, x^{\prime \prime} \in\left[y_{k}^{\prime}, \bar{x}_{k}\right)$, and suppose $y_{k}^{\prime \prime} \in\left(\bar{x}_{k-1}, y_{k}^{\prime}\right)$ such that

$$
\left|g\left(x^{\prime}\right)-g\left(x^{\prime \prime}\right)\right|<\epsilon / 8 \bar{n} M \quad \text { for } x^{\prime}, x^{\prime \prime} \in\left(\bar{x}_{k-1}, y_{k}^{\prime \prime}\right] .
$$

Let $\tilde{P}=\bar{P} \cup\left\{y_{1}^{\prime}, y_{2}^{\prime}, \cdots, y_{\bar{n}}^{\prime}, y_{1}^{\prime \prime}, y_{2}^{\prime \prime}, \cdots, y_{n^{\prime \prime}}{ }^{\prime}\right\}$. Let (1.1) be any partition of $[a, b]$ which is a refinement of $\tilde{P}$, and suppose $\zeta_{i} \in\left(x_{i-1}, x_{i}\right)$ for $i=1,2, \cdots, n$. For each $k=0,1,2, \cdots, \bar{n}$, let $i_{k}$ be the integer such that $0 \leqq i_{k} \leqq n$ and $x_{i_{k}}=\bar{x}_{k}$. Then 


$$
\begin{aligned}
& \mid\left\{g(a)\left[f\left(a^{+}\right)-f(a)\right]+g(b)\left[f(b)-f\left(b^{-}\right)\right]+\sum_{i=1}^{n-1} g\left(x_{i}\right)\left[f\left(x_{i}^{+}\right)-f\left(x_{i}^{-}\right)\right]\right. \\
& \left.+\sum_{i=1}^{n} g\left(\zeta_{i}\right)\left[f\left(x_{i}^{-}\right)-f\left(x_{i-1}^{+}\right)\right]\right\} \\
& -\sum_{i=1}^{n}\left[g\left(x_{i-1}\right)-g\left(\zeta_{i}\right)+g\left(x_{i}\right)\right]\left[f\left(x_{i}\right)-f\left(x_{i-1}\right)\right] \mid \\
& =\mid \sum_{i=1}^{n}\left\{\left[g\left(\zeta_{i}\right)-g\left(x_{i-1}\right)\right]\left[f\left(x_{i}\right)-f\left(x_{i-1}^{+}\right)\right]\right. \\
& \left.+\left[g\left(\zeta_{i}\right)-g\left(x_{i}\right)\right]\left[f\left(x_{i}^{-}\right)-f\left(x_{i-1}\right)\right]\right\} \\
& \leqq \sum_{k=1}^{\bar{n}}\left\{\left|g\left(\zeta_{i_{k}}\right)-g\left(x_{i_{k-1}}\right)\right|\left|f\left(x_{i_{k}}\right)-f\left(x_{i_{k}-1}^{+}\right)\right|\right. \\
& \left.+\left|g\left(\zeta_{i_{k-1}+1}\right)-g\left(x_{i_{k-1}+1}\right)\right|\left|f\left(x_{i_{k-1}+1}\right)-f\left(x_{i_{k-1}}\right)\right|\right\} \\
& +\sum_{k=1}^{\bar{n}}\left\{\sum_{i=i_{k-1}+1}^{k}\left|g\left(\zeta_{i}\right)-g\left(x_{i-1}\right)\right|\left|f\left(x_{i}\right)-f\left(x_{i-1}^{+}\right)\right|\right. \\
& \left.+\sum_{i=i_{k-1}+2}^{i_{k}}\left|g\left(\zeta_{i}\right)-g\left(x_{i}\right)\right|\left|f\left(x_{i}^{-}\right)-f\left(x_{i-1}\right)\right|\right\} \\
& \leqq 2\left[\bar{n} \frac{\epsilon}{8 \bar{n} M} 2 M\right]+\frac{\epsilon}{2\{V(g,[a, b])+1\}} \\
& \cdot \sum_{k=1}^{\bar{n}}\left\{\sum_{i=i_{k-1}+1}^{i_{k}^{-1}}\left|g\left(\zeta_{i}\right)-g\left(x_{i-1}\right)\right|+\sum_{i=i_{k-1}+2}^{i_{k}}\left|g\left(x_{i}\right)-g\left(\zeta_{i}\right)\right|\right\}<\epsilon .
\end{aligned}
$$

The weighted refinement integral (3.9) exists by Theorems 2.3 and 3.1. By the preceding paragraph, the Young refinement integral (1.9) exists and equals (3.9).

We conclude by generalizing MacNerney's result mentioned above for the case where the function $f$ is of bounded variation on $[a, b]$. First we establish directly the following result concerning the Young refinement integral (1.9) and the interior refinement integral (1.10) when $f$ is a saltus function on $[a, b]$.

THEOREM 3.7. Suppose $f$ is a saltus function and $g$ is bounded on $[a, b]$. Then (1.9) and (1.10) exist and the formula (1.8) holds.

Proof. Let $\left\{\bar{x}_{j}\right\}_{j=1}^{+\infty}$ be a sequence of distinct points of $(a, b)$ containing all points of $(a, b)$ where $f$ is discontinuous. Let $M$ be a posi- 
tive real number such that $|g(x)| \leqq M$ for $x \in[a, b]$.

The Young refinement integral (1.9) exists and equals

$$
g(a)\left[f\left(a^{+}\right)-f(a)\right]+g(b)\left[f(b)-f\left(b^{-}\right)\right]+\sum_{j=1}^{+\infty} g\left(\bar{x}_{j}\right)\left[f\left(\bar{x}_{j}^{+}\right)-f\left(\bar{x}_{j}^{-}\right)\right] .
$$

Suppose $\epsilon>0$. Let $\bar{n}$ be a positive integer such that

$$
\begin{aligned}
\mid g(a)\left[f\left(a^{+}\right)-f(a)\right] & +g(b)\left[f(b)-f\left(b^{-}\right)\right] \\
& +\sum_{j=1}^{\bar{n}} g\left(\bar{x}_{j}\right)\left[f\left(\bar{x}_{j}^{+}\right)-f\left(\bar{x}_{j}^{-}\right)\right]-\mathrm{FY} \int_{a}^{b} g(x) d f(x) \mid<\frac{\epsilon}{3}
\end{aligned}
$$

and such that

$$
\sum_{j=n+1}^{+\infty}\left|f\left(\bar{x}_{j}^{+}\right)-f\left(\bar{x}_{j}\right)\right|+\sum_{j=n_{+1}^{+1}}^{+\infty}\left|f\left(\bar{x}_{j}\right)-f\left(\bar{x}_{j}^{-}\right)\right|<\frac{\epsilon}{3 M} .
$$

Let $\bar{P}=\left\{a=\bar{\gamma}_{0}<\bar{\gamma}_{1}<\cdots<\bar{\gamma}_{\bar{n}+1}=b\right\}$ be $\left\{a, b, \bar{x}_{1}, \bar{x}_{2}, \cdots, \bar{x}_{n}\right\}$. Let $\tilde{P}$ be a partition of $[a, b]$ which is a refinement of $\bar{P}$ and is such that for each $j=1,2, \cdots, \bar{n}+1$ there are a $y_{j}^{\prime} \in\left(\bar{\gamma}_{j-1}, \bar{\gamma}_{j}\right) \cap \tilde{P}$ such that $\left|f(x)-f\left(\bar{\gamma}_{j-1}^{+}\right)\right|<\epsilon / 6(\bar{n}+1) M$ for $x \in\left(\bar{\gamma}_{j-1}, y_{j}^{\prime}\right)$ and a $y_{j}^{\prime \prime} \in\left(y_{j}^{\prime}, \bar{\gamma}_{j}\right)$ such that $\left|f(x)-f\left(\bar{\gamma}_{j}^{-}\right)\right|<\epsilon / 6(\bar{n}+1) M$ for $x \in\left(y_{j}^{\prime \prime}, \bar{\gamma}_{j}\right)$. Let (1.1) be a partition of $[a, b]$ which is a refinement of $\widetilde{P}$, and suppose $\zeta_{i} \in\left(x_{i-1}, x_{i}\right)$ for $i=1,2, \cdots, n$. For each $j=0,1, \cdots, \bar{n}+1$ let $i_{j}$ be the integer such that $0 \leqq i_{j} \leqq n$ and $x_{i_{j}}=\bar{\gamma}_{j}$. Let $T$ be the set of all positive integers $i$ less than $n$ such that $x_{i} \neq \bar{\gamma}_{j}$ for every $j=1,2, \cdots, \bar{n}$. Then

$$
\begin{aligned}
& \left|\sum_{i=1}^{n} f\left(\zeta_{i}\right)\left[g\left(x_{i}\right)-g\left(x_{i-1}\right)\right]-\left\{f(b) g(b)-f(a) g(a)-\mathrm{FY} \int_{a}^{b} g(x) d f(x)\right\}\right| \\
& =\mid-\sum_{j=1}^{\bar{n}+1} g\left(\bar{\gamma}_{j-1}\right)\left[f\left(\zeta_{i_{j-1}+1}\right)-f\left(\bar{\gamma}_{j}^{+}\right)\right]-\sum_{j=1}^{\bar{n}+1} g\left(\bar{\gamma}_{j}\right)\left[f\left(\bar{\gamma}_{\bar{j}}\right)-f\left(\zeta_{i_{j}}\right)\right] \\
& \quad-\sum_{i \in T} g\left(x_{i}\right)\left[f\left(\zeta_{i+1}\right)-f\left(\zeta_{i}\right)\right]+\sum_{j=n+1}^{+\infty} g\left(\bar{x}_{j}\right)\left[f\left(\bar{x}_{j}^{+}\right)-f\left(\bar{x}_{j}^{-}\right)\right] \mid \\
& <2 M(\bar{n}+1) \cdot \frac{\epsilon}{6(\bar{n}+1) M}+M\left\{\sum_{j=n+1}^{+\infty}\left|f\left(\bar{x}_{j}^{+}\right)-f\left(\bar{x}_{j}\right)\right|\right. \\
& \left.+\sum_{j=n+1}^{+\infty}\left|f\left(\bar{x}_{j}\right)-f\left(\bar{x}_{j}\right)\right|\right\}+\frac{\epsilon}{3}<\epsilon .
\end{aligned}
$$

Thus (1.10) exists and (1.8) holds.

Using Theorems 3.4 and 3.7, as well as integration-by-parts for the 
Riemann-Stieltjes norm integral, we have our final result.

THEOREM 3.8. Suppose $f$ is of bounded variation and $g$ is bounded on $[a, b]$. Then, the Young refinement integral (1.9) exists if and only if the interior refinement integral (1.10) exists, and in this case the formula (1.8) holds.

\section{REFERENCES}

1. H. L. Smith, On the existence of the Stieltjes integral, Trans. Amer. Math. Soc. 27 (1925), 491-515.

2. R. E. Lane, The integral of a function with respect to a function, Proc. Amer. Math. Soc. 5 (1954), 59-66.

3. B. Dushnik, Unpublished Ph.D. thesis, University of Michigan, Ann Arbor, Michigan, 1931.

4. J. S. MacNerney, An integration-by-parts formula, Bull. Amer. Math. Soc. 69 (1963), 803-805.

5. K. P. Smith and F. M. Wright, Existence conditions for the Lane integral, Proc. Amer. Math. Soc. 18 (1967), 1067-1073.

6. Henryk Schärf, Ueber links- und rechtsseitige Stieltjesintegrale und deren Anwendungen, Portugal. Math. 4 (1943), 73-118.

Iowa State University 